\title{
Structure of the Full-Length TRPV2 Channel by Cryo-EM
}

Kevin W. Huynh ${ }^{1}$, Matthew R. Cohen ${ }^{1,2}$, Jiansen Jiang ${ }^{4,5}$, Amrita Samanta ${ }^{1,2}$, David T. Lodowski ${ }^{1,3}$, Z. Hong Zhou ${ }^{4,5}$, Vera Y. Moiseenkova-Bell ${ }^{1,2}$

${ }^{1}$ Department of Pharmacology, School of Medicine, Case Western Reservere University, Cleveland, Ohio 44106, USA

${ }^{2}$ Department of Physiology and Biophysics, School of Medicine, Case Western Reservere University, Cleveland, Ohio 44106, USA

${ }^{3}$ Department of Nutrition, School of Medicine, Case Western Reservere University, Cleveland, Ohio 44106, USA

${ }^{4}$ Department of Microbiology, Immunology and Molecular Genetics, University of California Los Angeles, Los Angeles, California 90095, USA

${ }^{5}$ California NanoSystems Institute, University of California Los Angeles, Los Angeles, California 90095, USA

Transient receptor potential (TRP) channels form a superfamily $\mathrm{Ca}^{2+}$-permeable cation channels that are essential for detection of chemical and physical stimuli [1]. In addition, dysfunction or abnormalities in the regulation of these channels can result in a variety of neurological diseases, cardiovascular diseases and cancers [2]. Due to their wide variety of cellular functions, there has been a great interest in studying the structure, function and regulation of TRP channels. Many of these channels can interact with multiple ligands through different binding sites. Therefore, studying the structures of these channels may provide insights into their diverse regulatory mechanisms.

Cryo-electron microscopy (cryo-EM) has recently developed into a vital structural biology technique for resolving structure of small macromolecules $(<1 \mathrm{MDa}$ in size) including TRP channels to near-atomic resolution. At near-atomic resolution, conformational changes of these channels can be observed, which can provide insights into their regulatory mechanisms. The capsaicin receptor, transient receptor potential vanilloid 1 (TRPV1) of the TRPV subfamily was the first TRP channel to be resolved to nearatomic resolution by cryo-EM. The structure revealed a two-gate pore configuration and a mechanism of channel activation by agonists through changes in the outer pore region $[3,4]$. Though homologous to TRPV1, other TRPV channels (TRPV2-6) are insensitive to TRPV1 activators including heat and vanilloids. To further understand the structure TRPV subfamily members, we determined the structure of full-length TRPV2, which shares approximately $50 \%$ sequence identity to TRPV1, at $\sim 4.5 \AA$ resolution by cryo-EM (see Fig. 1).

Like TRPV1, TRPV2 contains two constrictions in the pore forming upper and lower gates. In the absence of activator, TRPV2 features wider upper and lower gates compared to the closed and the agonist-activated TRPV1. It is possible that the flexible loop connecting the S5 domain to the pore helix known as the pore turret, which was truncated in TRPV1 to stabilize the protein for structural studies, may account for these observed differences in the pore architecture. The pore turret has been shown to play a role in large cation permeation and pore dilation in TRP channels [5]. Interestingly, calcium responses to a TRPV2 activator 2-aminoethoxydiphenyl borate (2-APB) were ablated in a neuronal cell line expressing the pore turret deleted form of TRPV2. This suggests that the pore turret in TRPV2 may be important for 2-APB induced allosteric opening of the channel gate. 
The cryoEM structure of the full-length TRPV2 in the absence of ligand has revealed a wider pore compared the truncated TRPV1 suggesting that either the two channels have different intrinsic pore conformations or that the pore turret, which was truncated in TRPV1, may be essential for gating. We propose that these newly revealed TRPV2 structural features contribute to the diversity of TRPV channels. Further studies are necessary to determine endogenous regulators and the conformational changes associated with activation of other TRPV channels.

\section{References:}

[1] Venkatachalam K., Montell C., "TRP channels", Annu Rev Biochem 76 (2007), pp 387-417.

[2] Smani T., Shapovalov G., Skryma R., Prevarskaya N., Rosado JA., "Functional and

physiopathological implications of TRP channels", Biochimca et Biophysica Acta, 1853 (2015), pp

1772-1782.

[3] Liao M., Cao E., Julius D., Cheng Y., "Structure of the TRPV1 ion channel determined by electron cryo-microscopy", Nature, 504 (2013), pp 107-112.

[4] Cao E., Liao M, Cheng Y., Julius D., " TRPV1 structure in distinct conformations reveal activation mechanisms", Nature, 504 (2013), pp 113-118.

[5] Zheng J., Ma L., "Structure and function of the thermoTRP channel pore", Curr Top Membr 74 (2014), pp 233-257.

a.
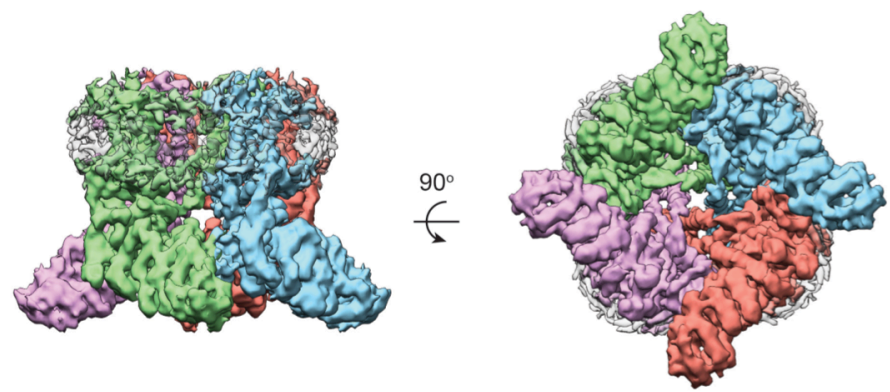

b.
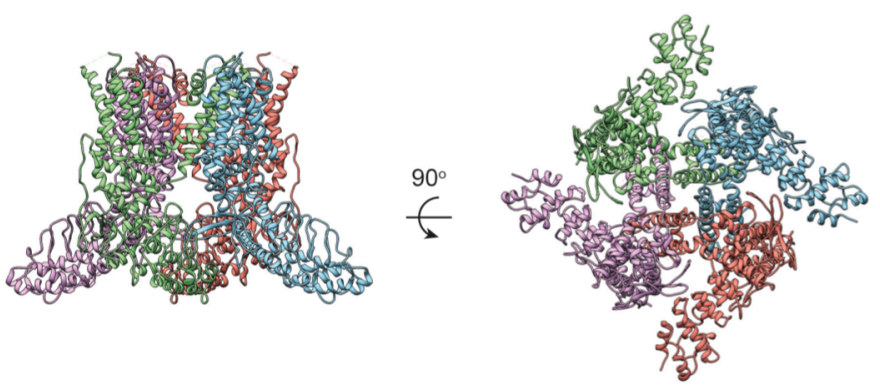

Fig. 1. Structure of full-length TRPV2. a. CryoEM density of TRPV2 shown from the side (left) and bottom (right) views with each subunit is represented by a different color. The transparent gray densities signify the detergent belt that stabilizes the hydrophobic regions of the channel. b. A model built from the cryoEM densities of TRPV2. The subunits are color in the same manner as the cryoEM densities. 